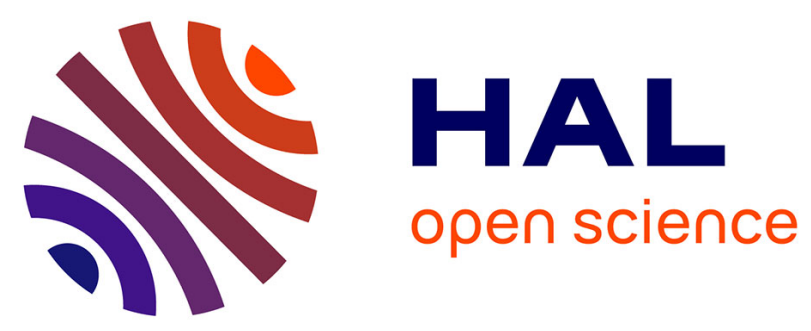

\title{
GYROSURFING ACCELERATION OF IONS IN FRONT OF EARTH's QUASI-PARALLEL BOW SHOCK
}

Arpad Kis, Oleksiy Agapitov, Vladimir Krasnoselskikh, Yuri V. Khotyaintsev, Iannis Dandouras, Istvan Lemperger, Viktor Wesztergom

\section{To cite this version:}

Arpad Kis, Oleksiy Agapitov, Vladimir Krasnoselskikh, Yuri V. Khotyaintsev, Iannis Dandouras, et al.. GYROSURFING ACCELERATION OF IONS IN FRONT OF EARTH's QUASI-PARALLEL BOW SHOCK. The Astrophysical journal letters, 2013, 771 (1), pp.4. 10.1088/0004-637X/771/1/4 . insu-01258093

\section{HAL Id: insu-01258093 \\ https://hal-insu.archives-ouvertes.fr/insu-01258093}

Submitted on 18 Jan 2016

HAL is a multi-disciplinary open access archive for the deposit and dissemination of scientific research documents, whether they are published or not. The documents may come from teaching and research institutions in France or abroad, or from public or private research centers.
L'archive ouverte pluridisciplinaire HAL, est destinée au dépôt et à la diffusion de documents scientifiques de niveau recherche, publiés ou non, émanant des établissements d'enseignement et de recherche français ou étrangers, des laboratoires publics ou privés. 


\title{
GYROSURFING ACCELERATION OF IONS IN FRONT OF EARTH's QUASI-PARALLEL BOW SHOCK
}

\author{
Arpad Kis ${ }^{1,5}$, Oleksiy Agapitov ${ }^{2,6}$, Vladimir Krasnoselskikh ${ }^{2}$, Yuri V. KhotyaintseV ${ }^{3}$, \\ IANNIS DANDOURAs ${ }^{4}$, ISTVAN LEMPERGER ${ }^{1}$, AND VIKTOR WESZTERGOM ${ }^{1}$ \\ ${ }^{1}$ Research Centre for Astronomy and Earth Sciences, Geodetic and Geophysical Institute, Sopron, Hungary; akis@ggki.hu, Kis.Arpad@csfk.mta.hu \\ ${ }^{2}$ LPC2E/CNRS, F-45071 Orleans, France \\ ${ }^{3}$ Swedish Institute of Space Physics, SE- 75121 Uppsala, Sweden \\ ${ }^{4}$ CESR, F-31028 Toulouse, France \\ Received 2012 September 11; accepted 2013 May 3; published 2013 June 7
}

\begin{abstract}
It is well known that shocks in space plasmas can accelerate particles to high energies. However, many details of the shock acceleration mechanism are still unknown. A critical element of shock acceleration is the injection problem; i.e., the presence of the so called seed particle population that is needed for the acceleration to work efficiently. In our case study, we present for the first time observational evidence of gyroresonant surfing acceleration in front of Earth's quasi-parallel bow shock resulting in the appearance of the long-suspected seed particle population. For our analysis, we use simultaneous multi-spacecraft measurements provided by the Cluster spacecraft ion (CIS), magnetic (FGM), and electric field and wave instrument (EFW) during a time period of large inter-spacecraft separation distance. The spacecraft were moving toward the bow shock and were situated in the foreshock region. The results show that the gyroresonance surfing acceleration takes place as a consequence of interaction between circularly polarized monochromatic (or quasi-monochromatic) transversal electromagnetic plasma waves and short large amplitude magnetic structures (SLAMSs). The magnetic mirror force of the SLAMS provides the resonant conditions for the ions trapped by the waves and results in the acceleration of ions. Since wave packets with circular polarization and different kinds of magnetic structures are very commonly observed in front of Earth's quasi-parallel bow shock, the gyroresonant surfing acceleration proves to be an important particle injection mechanism. We also show that seed ions are accelerated directly from the solar wind ion population.
\end{abstract}

Key words: acceleration of particles - plasmas - shock waves - supernovae: general - waves

Online-only material: color figures

\section{INTRODUCTION}

There exist many astrophysical objects and systems where strong shocks are present. Supernova remnants, active galactic nucleus jets, pulsar wind nebulae, and gamma-ray bursts are some of the astrophysical objects where a shock wave is supposed to be formed and is accompanied by a swarm of energetic particles producing radiation. It is widely accepted that the major mechanism responsible for particle acceleration by shock waves and which is capable of explaining the main characteristics of the formed particle distribution, such as the power-law energy spectra, is diffusive shock acceleration (DSA), a specific form of first-order Fermi acceleration. The mechanism is basically simple, since the energy gain occurs by means of the bouncing of particles between the upstream and downstream regions of the shock front and the energy gain is due to the converging plasma flows in these regions separated by the shock front. The success of this model lies in its ability to provide a quite high efficiency of energy transfer and in its universality. The basic ideas were proposed after 1970 by Krymskii (1977), Axford et al. (1978), Bell (1978a, 1978b), and Blandford \& Ostriker (1978). There are three important conditions to be satisfied in order to ensure the high efficiency of the mechanism operation (Malkov \& O'C Drury 2001): (1) some initial "seed" population should be created upstream or downstream of the shock that should be able to cross the shock front, (2) there should exist scattering centers or objects around the shock front in its vicinity that will deviate particles from their free motion

\footnotetext{
5 Visiting Scientist at LPC2E/CNRS Orleans, France.

${ }^{6}$ National Taras Shevchenko University of Kiev, Ukraine.
}

and will result in returning them to the opposite direction, and (3) the pressure of energetic particles should not smear out the shock front transition. In non-relativistic and weakly relativistic shocks, it is suggested that the particles scatter on Alfvén waves generated by the same energetic particle population. Such a self-consistent pattern can be well described for a highenergy population that is formed in the frame of quasi-linear approximation. However, this process requires the injection of the lower energy suprathermal population that should feed this process. The process of the formation of a relatively lowenergy suprathermal population of energetic particles that can become the "seed" population for the DSA was addressed in many theoretical works as well as in different simulations. An important question is whether this process is universal and similar at all types of shocks or not. This question cannot be answered today due to the lack of information about relativistic and ultrarelativistic shocks. However, there exist quite important similarities in parameters between supernova remnant shocks and planetary bow shocks in the heliosphere. One can use direct in situ measurements provided by satellites to study different populations of energetic suprathermal particles and their relation to different wave structures observed in the vicinity of the planetary or Earth bow shock. It is known that the most efficient accelerator is the quasi-parallel shock, where the conditions for the DSA mechanism are the most favorable. The structure of the quasi-parallel shock was studied in detail by making use of direct in situ measurements on board numerous satellites. A widely accepted pattern of the shock front structure is suggested to consist of a long transition region filled by many magnetic structures, the so called SLAMS (short large amplitude magnetic structure). The name was introduced by Schwartz \& Burgess 
(1991), who analyzed magnetic field data on board AMPTE satellites. These structures can be either isolated or immersed in smaller amplitude wave activity. Giacalone et al. (1993) have classified these two groups as isolated and embedded SLAMS. It was suggested by Scholer et al. (1992) that SLAMS can grow from ultra-low frequency (ULF) wave packets passing through a local energetic particle gradient. It was noted that SLAMS, during their convective motion toward the shock front, presumably sweep up the energetic ion population. An important and often-observed interesting feature of embedded SLAMS is the precursor wave train that means quite often quasimonochromatic wave packets that can span for a long distance in front of the SLAMS. These precursor wave trains are often cited as $1 \mathrm{~Hz}$ waves (Russell 1988). The interaction between such a wave precursor and a magnetic or electric increasing field profile was found to be an efficient particle accelerator (Kuramitsu \& Krasnoselskikh 2005a and Kuramitsu \& Krasnoselskikh 2005b); this acceleration mechanism is known as the gyrosurfing acceleration (GSA). The mechanism itself is a very simple one: ions can be trapped by the wave which has a frequency close to their gyrofrequency in the reference frame moving with them. In such a situation, the parallel velocity of the trapped ions oscillates around the resonance velocity; no energy gain by the ions can be observed. On the other hand, in the presence of an external force (like a changing electrostatic profile that interacts with the trapped ions), the ions can be kept in the exact resonance condition, and thus they can be accelerated by the electric field of the wave monotonically in the perpendicular direction. Since monochromatic wave packets with circular polarization and different kinds of magnetic structures are very commonly observed in the front of Earth's quasi-parallel bow shock, the gyroresonant surfing acceleration might prove to be an important particle injection mechanism that is capable of producing the seed ions in abundance. The aim of our paper is to study this acceleration mechanism in front of Earth's quasiparallel shock and to present observational evidence that this acceleration results in the energization of "seed" ions in the close vicinity of large amplitude magnetic structures.

\section{OBSERVATIONS}

For this study we have chosen the time period of 2003 February 18, when the Cluster spacecraft were located upstream of Earth's quasi-parallel bow shock (i.e., in the solar wind) and they were moving toward the shock. The inter-spacecraft separation distance was rather large, varying from $\sim 1 R_{E}$ to $\sim 1.5 R_{E}$. The average interplanetary magnetic field orientation was rather constant, providing a continuous connection to the quasi-parallel side of the bow shock for more than $10 \mathrm{hr}$. The solar wind speed was high, about $600 \mathrm{~km} \mathrm{~s}^{-1}$, and the Alfvén Mach number was close to 8 . The solar wind density varied between 2.4 and $7 \mathrm{~cm}^{-3}$ on average. All these plasma parameters show that at the time of the observation, there was a high solar wind velocity and high Mach number shock, and the steady-state conditions for the forming of the bow shock and the foreshock region existed; no sudden or abrupt, large-scale changes in the interplanetary conditions were recorded. For our analysis, we used magnetic and ion data provided by the Cluster spacecraft. The high resolution (i.e., $5 \mathrm{~Hz}$ sampling frequency) magnetic data were obtained by the FluxGate Magnetometer (Balogh et al. 2001). The particle data were provided by the Cluster Ion Spectrometer, which consists of the Hot Ion Analyzer (HIA) and the COmposition and DIstribution Function analyzer (Rème et al. 2001). We used the data from HIA because this instrument
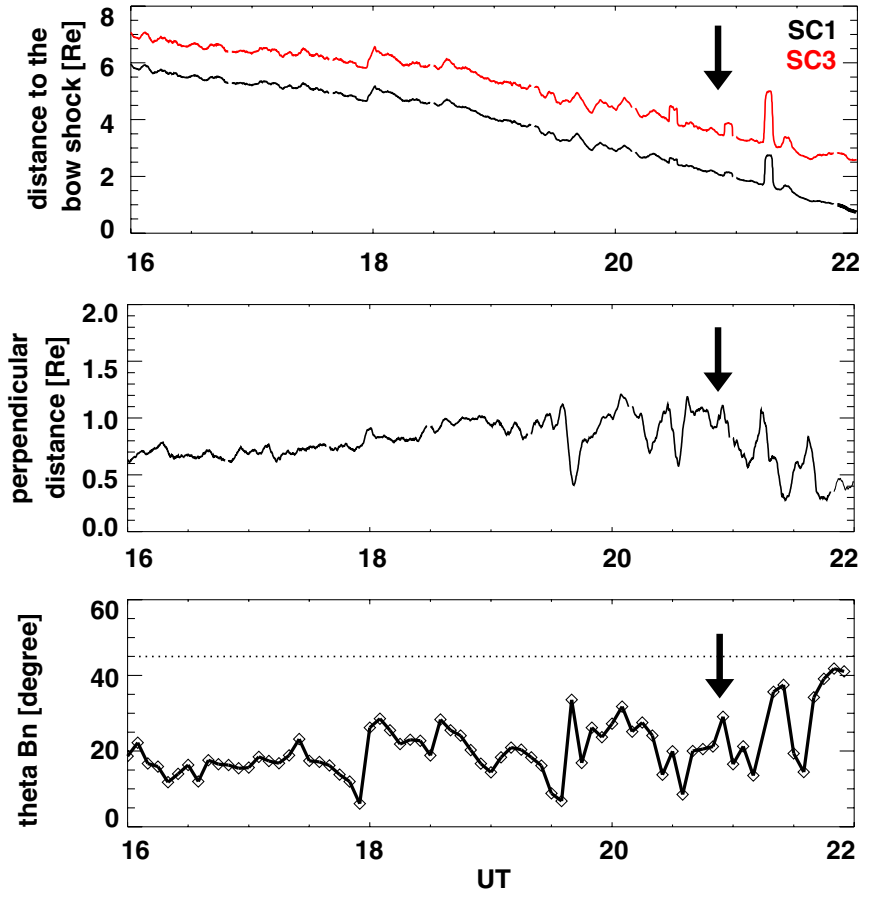

Figure 1. Upper panel presents the distance of SC1 (black) and SC3 (red) to the bow shock along the magnetic field line; it can be observed that SC1 was situated for the entire time closer to bow shock, while SC3 was situated further upstream. The middle panel shows the distance between the two spacecraft in the perpendicular direction (i.e., related to the direction of the local magnetic field). The lower panel presents the angle between the local magnetic field and the bow shock normal direction. The horizontal punctuated line marks the $\theta_{B n}$ value of $45^{\circ}$. The black arrow marks, in all three panels, the time period of the detailed analysis when the seed particle population was recorded.

(A color version of this figure is available in the online journal.)

provides partial ion density data in the $10-13 \mathrm{keV}, 13-18 \mathrm{keV}$, 18-24 keV, and 24-32 keV ion energy ranges. In our analysis, we used particle data from SC1 and SC3; the reason for this is that during the time period of investigation, there is no HIA data from SC2 and SC4. On the other hand, the availability of magnetic data from all four spacecraft and the optimal location of SC1 and SC3, combined with the large separation distance between them, provides the ideal conditions to perform the analysis. In order to determine the SC distance to the bow shock along the magnetic field line, we used the bow shock model by Peredo et al. (1995). This bow shock model provides a normalized three-dimensional bow shock shape depending on the Mach number. The shock surface normal direction given by this model agrees very well with normal directions which were determined by using in situ direct measurements (Horbury et al. 2001). This modeled bow shock surface was adapted to the actual bow shock position using the exact location of SC1 at the time when the spacecraft recorded the bow shock crossing. This expansion then was adapted to the $10 \mathrm{hr}$ time period before the crossing by taking into account the variations in the solar wind pressure. By using this scaled and dynamic bow shock model, we determined the distance of Cluster SC to the bow shock along the magnetic field line. In addition, the analysis also provides the angle $\theta_{B n}$ between the shock normal and the magnetic field at the intersection point of the magnetic field and the bow shock surface. Figure 1 presents three panels. The upper panel shows the distance of SC1 (black line) and SC3 (red line) to the bow shock along the magnetic field line as a function of time. It can be observed that SC1 was situated closer to the bow shock for the entire period and SC3 was 


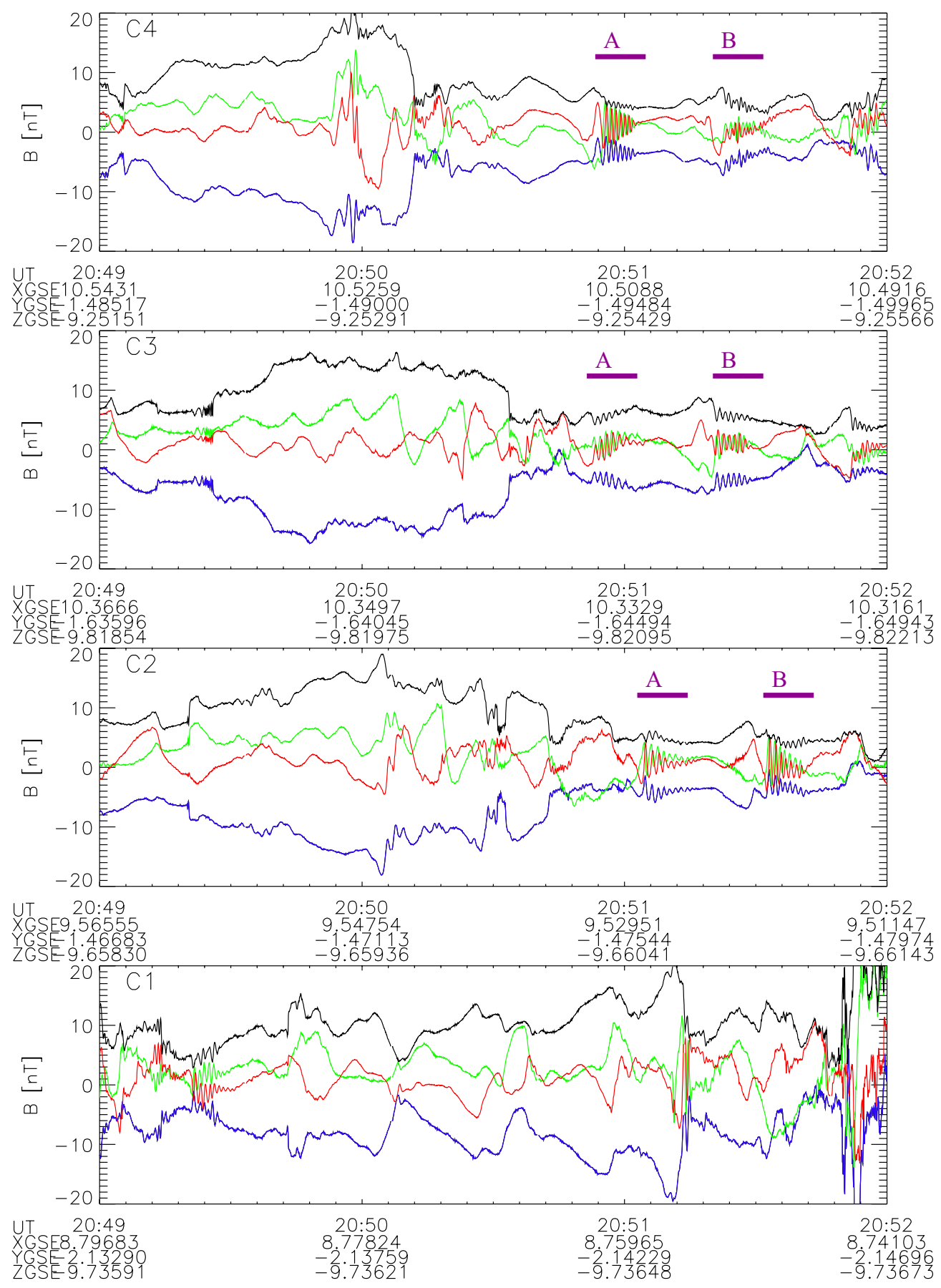

Figure 2. Magnetic field components (shown in colours) and the magnetic field absolute value (shown in black) observed by the four spacecraft between $20: 49$ and 20:52 UT. The top panel in the figure presents the magnetic field recorded by SC4 (which was the farthest situated from the bow shock), while the bottom panel presents the magnetic field recorded by SC1 (which was the closest located spacecraft to the bow shock); the magnetic field recorded by SC3 and SC2 are presented in the middle two panels. The wave packets can be easily observed in the magnetic data recorded by SC2, SC3, and SC4. Horizontal magenta bars indicate the time periods when the two wave packets were observed; the first one (in chronological order) is marked A, while the second one is marked B.

(A color version of this figure is available in the online journal.)

situated further upstream while both were moving closer to the bow shock. The distance between the two spacecraft parallel to the magnetic field varies between 1 and $1.5 R_{E}$. The difference in distance of $\mathrm{SC} 1$ and $\mathrm{SC} 3$ to the bow shock provides the necessary spacial resolution for the simultaneous multi-point ion and magnetic measurements recorded in the foreshock region. The fluctuations in the distance of the two SC to the bow shock is due to small-scale variations in the magnetic field, and in the solar wind density and velocity. The black arrow marks the time when the seed particle population was observed (this is described in more detail in the following). The middle panel presents the distance between $\mathrm{SC} 1$ and $\mathrm{SC} 3$ in the direction perpendicular to the local magnetic field. It can be observed that this perpendicular distance varies between 0.5 and $1 R_{E}$ on average. Since the Larmor radius of the observed upstream ions is comparable with the value of the distance between the two SC in the perpendicular direction, we can conclude that $\mathrm{SC} 1$ and $\mathrm{SC} 3$ observe ion populations connected to the 

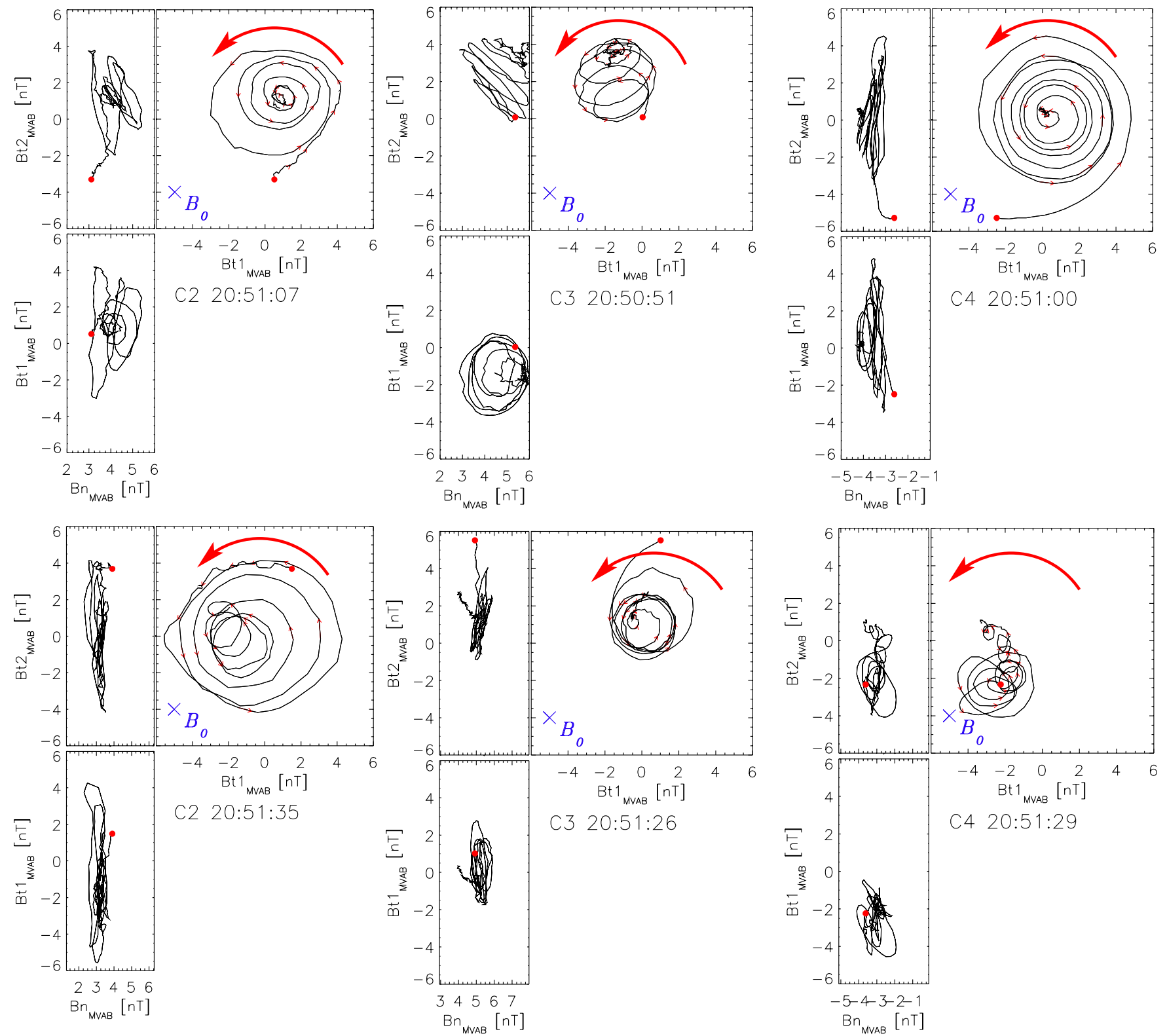

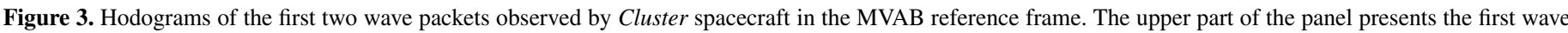

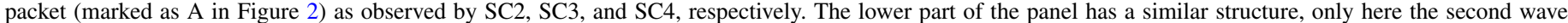

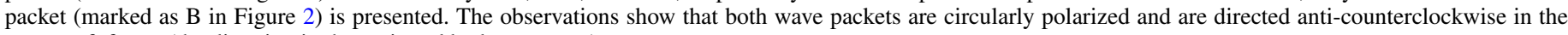
spacecraft frame (the direction is shown in red by bent arrows).

(A color version of this figure is available in the online journal.)

same magnetic field line. Therefore, any difference observed in the ion distribution functions by $\mathrm{SC} 1$ and $\mathrm{SC} 3$ can only be interpreted as differences due to the spatial evolution of the ion distribution and composition as a function of the distance to the quasi-parallel bow shock. Finally, the lower panel presents the dynamics of $\theta_{B n}$ at the intersection point of the bow shock surface with the magnetic field connected to the SC versus time. We can observe that the value of $\theta_{B n}$ for the whole time period remains far below $45^{\circ}$ (marked with the dashed horizontal line), which indicates that the SC are situated deep in the foreshock region (i.e., on the quasi-parallel side). Here, we present only one line because $\theta_{B n}$ is determined by using the magnetic field recorded at $\mathrm{SC} 1$ and $\mathrm{SC} 3$, which match each other quite well and therefore are overlapping. For the detailed analysis we have chosen a $180 \mathrm{~s}$ time period from 20:49 to
20:52 UT. At this time, SC1 was situated at a distance of $\sim 2 R_{E}$ from the bow shock (in the direction of the magnetic field), while SC3 was situated further upstream at a distance of $\sim 3.5 R_{E}$ from the shock. SC4 was located even further upstream from SC3, while SC2 was between $\mathrm{SC} 1$ and SC3. The location of the four spacecraft and their various distances from the bow shock created ideal conditions to investigate in detail the temporal and spatial evolution of the space plasma characteristics. Figure 2 presents the magnetic field components observed by the four spacecraft in the three-minute time interval. The top panel in the figure presents the magnetic field recorded by SC4 (which was the farthest situated from the bow shock), while the bottom panel presents the magnetic field recorded by $\mathrm{SC} 1$ (which was the spacecraft located closest to the bow shock). According to ACE, WIND, and Cluster measurements, 


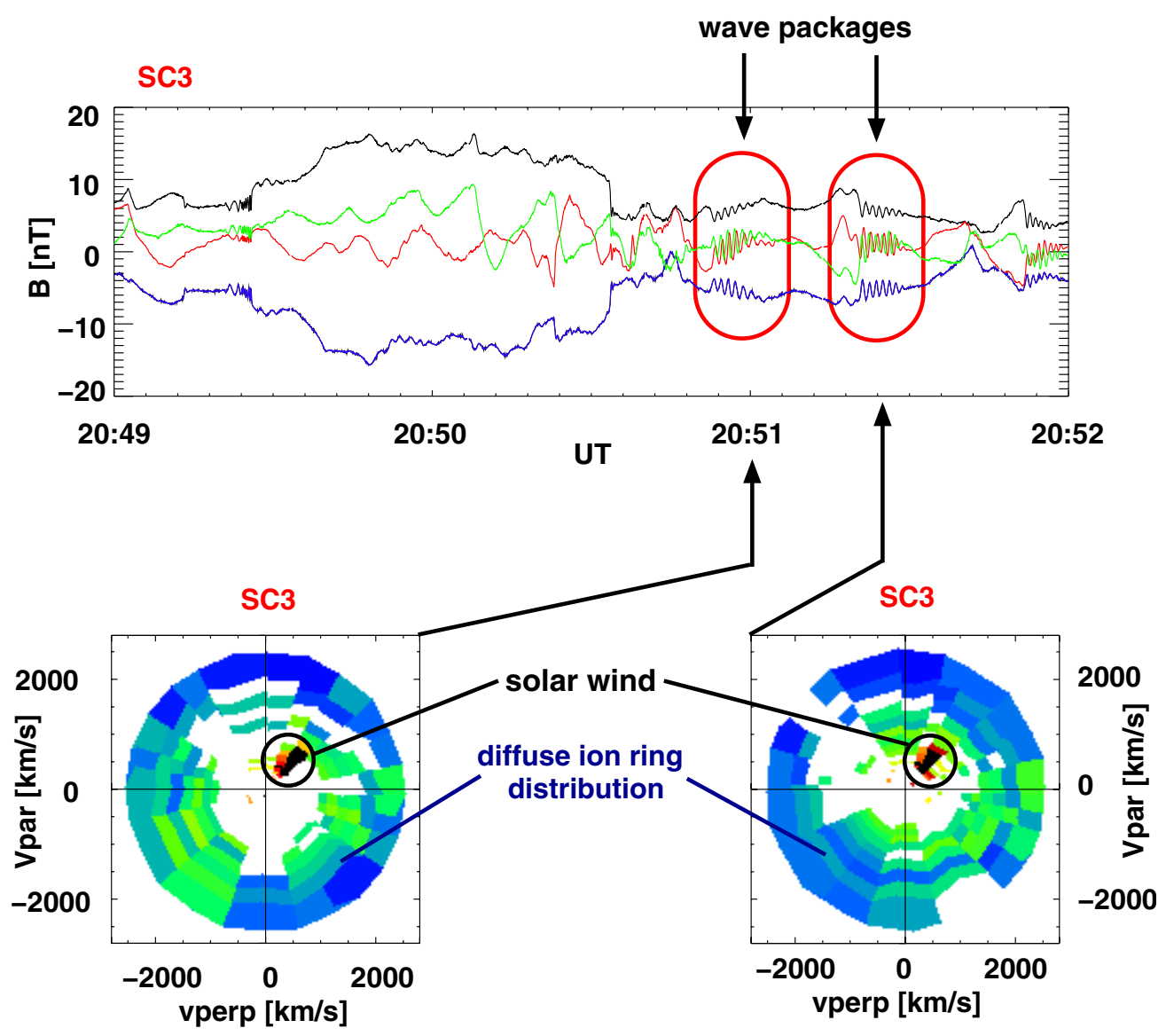

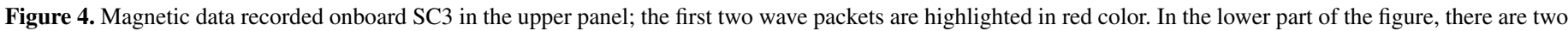

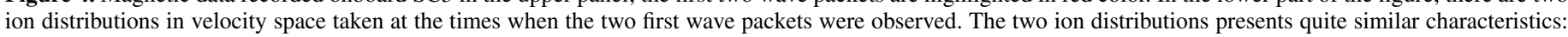
the highly isotropic ring of the diffuse ions can be observed together with the marked beam-like distribution of the solar wind.

the average values (during the three-minute time period) of the solar wind velocity, interplanetary magnetic field, magnetic field normal vector, and ion density was $V_{\mathrm{SW}} \simeq 600 \mathrm{~km} \mathrm{~s}^{-1}$, $|B|=6.6 \mathrm{nT}(-4.4,-0.12,1.44), n_{B}=(-0.95,-0.03,0.31)$, and $n_{i}=4 \mathrm{~cm}^{-3}$, respectively. The solar wind ion gyrofrequency $f_{i}$ was $\sim 0.1 \mathrm{~Hz}$. Three wave packets were detected during the time interval from 20:50 to 20:52 UT. The characteristics of these waves were analyzed by using the minimum variance analysis of magnetic field perturbations (MVAB) technique for the wave-normal vector $\boldsymbol{k}$ evaluation. The wave polarization is determined in the reference frame, where one of the axes is chosen along wave normal and the others according to the wave polarization ellipse axes. In Figure 2, the three wave packets can be clearly observed on the magnetic field records provided by SC4 and SC3; the magnetic data provided by SC2 only shows the first two waves: this is due to the time shift between SC as a consequence of the convection by the solar wind. The first wave packet (in chronological order) was recorded by SC3 at 20:50:53 and by SC4 at 20:50:55 UT (that is consistent with wavevector direction obtained by MVAB). The same wave packet was observed onboard C2 at 20:51:04 UT. At SC1's location, these wave packets cannot be seen anymore because these waves are merging/already merged with the magnetic structure. Since the intensity (i.e., amplitude) of the magnetic structure is almost one order of magnitude larger than the wave amplitude and close to the bow shock, the magnetic environment becomes more and more turbulent and the amplitude of the magnetic structure is increased, its shape changed, and the wave packet(s) cannot be distinguished clearly anymore. All three wave packages were identified as magnetosonic waves with circular polarization. Wave polarization in the MVAB reference frame is shown in Figure 3. It is anti-counterclockwise (wave magnetic field vector rotates in the same direction as ions rotate in the magnetic field) in the spacecraft reference frame but counterclockwise in the solar wind frame. Thus, the wave can affect ions with velocities less than the wave phase velocity in the spacecraft frame. Since the wave packets were observed by all spacecraft, and taking into account the spacecraft position and also the convection by the solar wind, the characteristics of these waves (direction and speed of propagation, etc.) can be determined. Wave amplitude changes transverse to wavevector direction with a spatial scale of about $1.5 R_{E}$. Wavevector directions were close to parallel related to the background magnetic field direction; the angle between the wave normal and the background magnetic field direction is about $10^{\circ}-20^{\circ}$. The wave front was found to be planar on the spatial scale of the inter-spacecraft distances. The wave frequency in the frame of the spacecraft is $f_{w}=$ $0.7-1.0 \mathrm{~Hz}$. The frequency of the waves in the solar wind frame was $\sim 0.2 \mathrm{~Hz}$ (greater than the ions gyrofrequency) and the wavelength was about $350-490 \mathrm{~km}$. Timing analysis gave the phase velocity of the waves the value of $490 \mathrm{~km} \mathrm{~s}^{-1}$ in the spacecraft reference frame. The time delay between the spacecraft observing the waves is close to the estimation of the local Alfvén velocity value $\left(V_{A}=80-100 \mathrm{~km} \mathrm{~s}^{-1}\right)$ for the waves which were propagating in the sunward direction in the solar wind frame. Besides the three wave packets, Figure 2 shows 


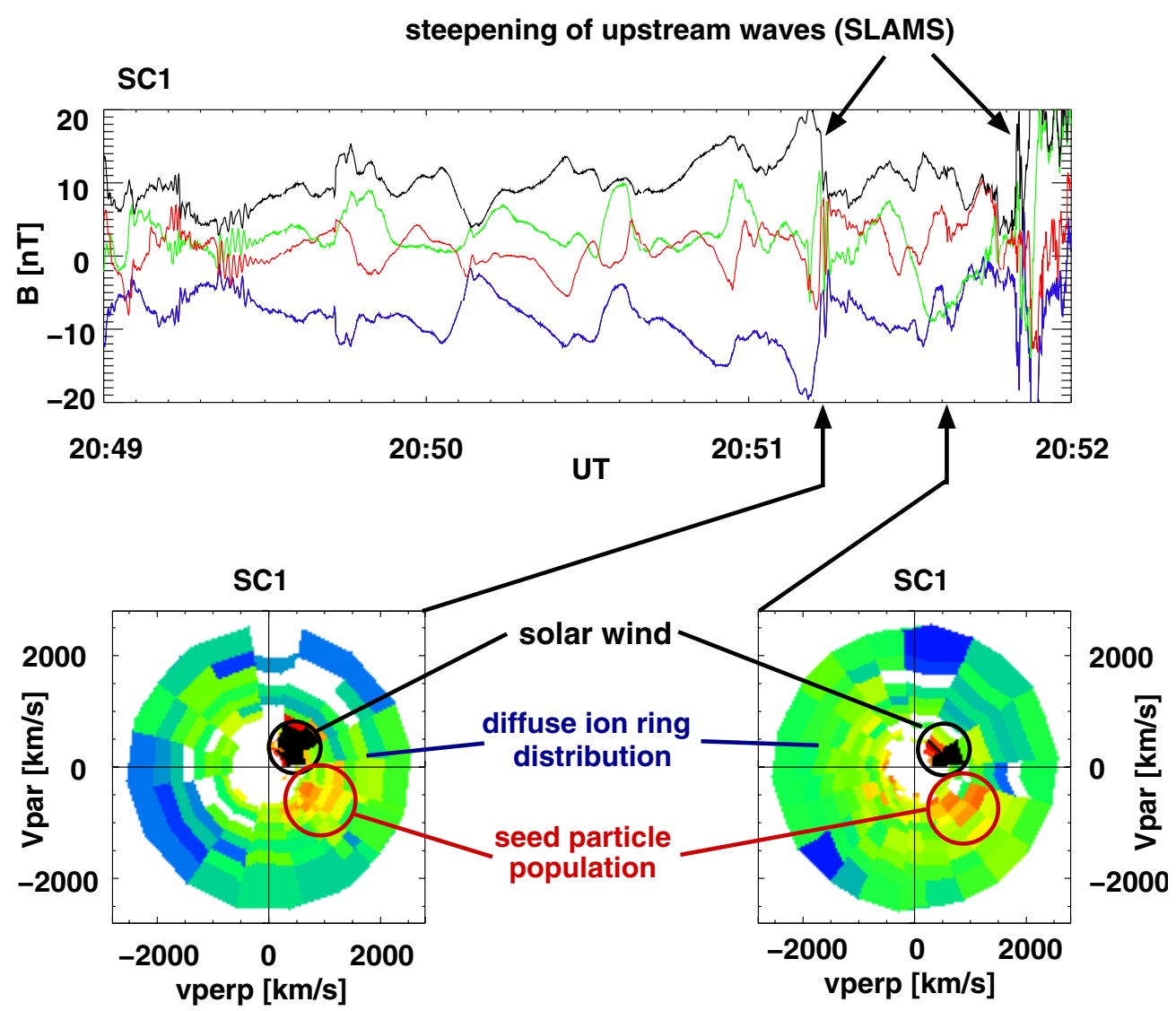

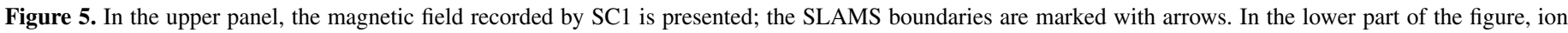

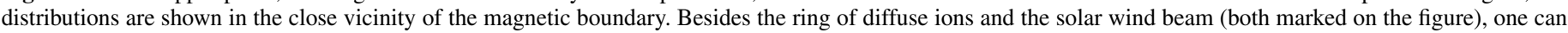

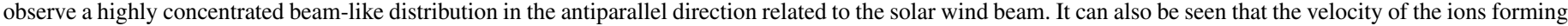
the beam is slightly higher than of the ions forming the solar wind beam; a typical characteristic of a seed ion population

a magnetic structure with a boundary that can be described as a sudden jump in the magnetic field value. This boundary was detected by all Cluster spacecraft: SC4 recorded this boundary at 20:50:11, SC3 at 20:50:34, SC2 at 20:50:45, and SC1 at 20:51:14 UT, respectively. The boundary moves along the direction $(-0.52,-0.49,-0.69)$ in GSE coordinates, with a normal velocity of about $164 \mathrm{~km} \mathrm{~s}^{-1}$. It can be seen that SC1 observes a steepening of this structure; this steepening is in accordance with the suggestion by Scholer et al. (1992) that SLAMS can grow from steepening ULF waves as they are convected closer to the bow shock. We have to emphasize that $\mathrm{SC} 1$ was in a favorable position to record the steepening of the magnetic structure (resulting in SLAMS) and at the same time the merging of the wave packet with the magnetic structure, which explains why the wave packet cannot be observed clearly anymore at the position of SC1. On the other hand, since the propagation of the direction and velocity of the wave packet and of the magnetic structure were determined from the data provided by the other three spacecraft, we can be certain that $\mathrm{SC} 1$ observes the merging of the wave packet with the magnetic structure that can be identified as a SLAMS. We would like to emphasize that the process of merging (or overlapping) of the waves with the magnetic structure takes place during a defined period of time, therefore the exact moment of the merging cannot be determined. This also means that by "merging" we understand a somewhat continuous process and between "before" and "after" there is a transition phase. In our analysis, we do not study the evolution of the amplitude or shape of the waves or of the magnetic structure since the study focuses on demonstrating the very existence of the GSA. On the other hand, it has to be mentioned that the evolution of the magnetic structures and waves as a function of distance from the bow shock might play an important role in determining the efficiency of the GSA mechanism.

To study the interaction of ions with the wave packets and the magnetic structure, we determine the ion distributions at critical time periods. Figure 4 presents the magnetic data recorded onboard SC3 in the upper panel of the figure; the first two wave packets are highlighted in red. In the lower part of the figure, there are ion distributions in velocity space taken at the times when the two first wave packets were observed. The two ion distributions present quite similar characteristics: the highly isotropic ring of the diffuse ions can be observed together with the marked beam-like distribution of the solar wind. The presence of diffuse ions in front of the quasi-parallel bow shock is a common and well known feature of the foreshock region. Figure 5 has a structure similar to Figure 4: on the upper panel, the magnetic field recorded by SC1 is presented. The SLAMS boundaries are marked with arrows. In the lower part of the figure, the ion distributions are presented. As was mentioned before, the waves packets observed by SC3 cannot be seen anymore due to the interaction with the magnetic structure boundaries. However, the ion distributions were taken at the time that was estimated by taking into consideration the wave packet velocity and propagation direction, and the convection of the waves by the solar wind and the SC position. 

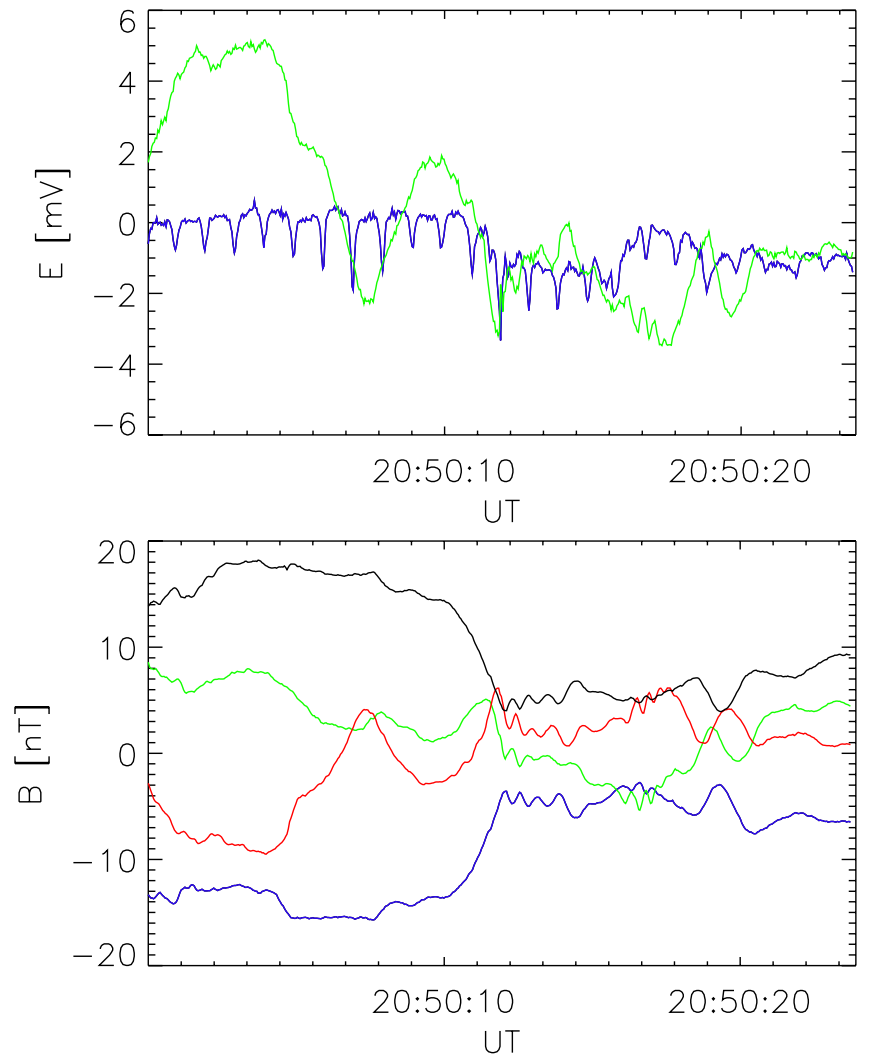

Figure 6. In the upper panel, the electric field $X$ (in blue) and $Y$ (in green) component values recorded by the EFW instrument onboard SC4 are presented. The units are in $\mathrm{mV} \mathrm{m}^{-1}$ at the time interval when the magnetic field structure was observed, which can be seen in the lower panel. It can be clearly observed that at the magnetic boundary there is no significant jump in the electric field value.

(A color version of this figure is available in the online journal.)

In other words, these distributions present the characteristics of the ion population at the time period when the wave packets interact (i.e., merge) with the magnetic structure. The ion distributions show the ring of diffuse ions and the concentrated beam of the solar wind; very similar to what was observed at SC3. However, the intensity of the diffuse ion ring is clearly higher than the intensity of the ion distributions that was recorded aboard SC3. This is in accordance with earlier observations since it is well known that the diffuse ion density is growing exponentially with decreasing distance from Earth's bow shock. Besides the ring of diffuse ions and the solar wind beam (both marked on the figure), one can observe a concentrated beam-like distribution in the antiparallel direction related to the solar wind. It can also be seen that the velocity of the ions forming the mentioned beam is slightly higher than the velocity of the solar wind ions; this means that the ions have a higher energy than solar wind ions. In other words, this ion beam shows all the characteristics that are typical for a seed ion population and is observed in the close vicinity of a wave packet that is merging with a magnetic structure.

\section{DISCUSSION}

In general, GSA can be efficient due to the combination of two effects: the trapping of particles by circularly polarized monochromatic electromagnetic waves and the presence of an external force that keeps the ions in the resonance condition. Using the results by Kuramitsu \& Krasnoselskikh (2005a), if the parallel component of the particle velocity is close to the resonant velocity, then the time derivative of the perpendicular energy becomes positive and the transverse component of the particle velocity is increased significantly. The resonance condition for the ions trapped by the waves is

$$
n \Omega_{i}=\boldsymbol{k} \boldsymbol{V}-\omega_{\mathrm{wave}},
$$

where $\boldsymbol{V}$ and $\omega_{\text {wave }}$ are the resonant ion velocity and wave frequency in the solar wind frame, respectively, and can be satisfied for cyclotron resonance conditions $n= \pm 1$ if the $\boldsymbol{V}$ component along the magnetic field is directed anti-sunward (i.e., in the same direction as the solar wind plasma flow) and is equal to $440-450 \mathrm{~km} \mathrm{~s}^{-1}$ for an anti-counterclockwise wave (which is observed in our case) or $530-540 \mathrm{~km} \mathrm{~s}^{-1}$ for a counterclockwise wave, respectively. In the absence of an appropriate external force, the Lorentz force of the wave breaks rapidly the resonance conditions for ions in a homogeneous system. To explain the observed acceleration of the ions due to transverse energy gain, we use the basic idea of gyroresonant surfing from Kuramitsu \& Krasnoselskikh (2005a), but in a somewhat modified form. The background electric field does not have any inhomogeneity and therefore cannot compensate the wave Lorentz force as it is discussed in Kuramitsu \& Krasnoselskikh (2005a). Figure 6 presents in the upper panel the electric field $X$ (in blue) and $Y$ (in green) component values recorded by the electric field and wave (EFW) instrument (Gustafsson et al. 2001; Khotyaintsev et al. 2010) on board SC4. The units are in $\mathrm{mV} \mathrm{m}^{-1}$ at the time interval when the magnetic field structure was observed, which can be seen in the lower panel. It can be clearly seen that at the magnetic boundary, there is no significant jump in the electric field value. However, in our case, the wave Lorentz force can be compensated by the inhomogeneity of the magnetic field. We consider the ion motion in the field of the electromagnetic wave, which propagates along the background magnetic field. In the absence of a background electric field, the equation of motion is

$$
m \frac{d \boldsymbol{V}(\boldsymbol{r}, t)}{d t}=\frac{e}{c}\left[\boldsymbol{V}(\boldsymbol{r}, t) \times\left(\boldsymbol{B}(x)+\boldsymbol{B}_{w}(\boldsymbol{r}, t)\right)\right]+e \boldsymbol{E}_{w}(\boldsymbol{r}, t),
$$

where $\boldsymbol{B}(x)=\left(B_{0}, B y, B z\right)$ is the background magnetic field, $\boldsymbol{B}_{w}(\boldsymbol{r}, t)$ and $\boldsymbol{E}_{w}(\boldsymbol{r}, t)$ are the wave magnetic and electric field, and $\boldsymbol{V}$ is the ion velocity. The $B_{0}$ is the background (i.e., average) magnetic field that also determines the $x$-axis direction. Following Krymskii (1977) but using the magnetic pressure gradient instead of the electric field, it is possible to keep $V_{x}$ constant and to hold the resonance condition with the wave. As has been mentioned before, the wave propagates without effective wave-particle interaction in a homogeneous system, but in the vicinity of a magnetic structure the Lorentz force of the wave can be balanced by the inhomogeneity of the magnetic field (i.e., of the SLAMS structure). Consequently, the gyrosurfing mechanism is realized and ions gain the energy in the perpendicular direction related to the background magnetic field. The resonance condition in a changing magnetic field changes slowly ( $\omega \ll k V_{x}+\Omega=0$ ), and as was shown by Kuramitsu \& Krasnoselskikh (2005a), the transverse energy gain is also effective near the resonance condition. It was also shown that if the trapping region becomes large as the wave amplitude becomes larger, and in the presence of large amplitude waves, even non-resonant but close to it particles can be trapped and accelerated. Because of this flexibility or non-dependence on the trapped ion velocity and motion, the gyroresonant surfing can work efficiently in the presence 
of a wider class of magnetic field dependencies on $x$, such as shock-like dependencies (Kuramitsu \& Krasnoselskikh 2005a). The ion transverse energy gain also leads to the increasing of the particle pitch angle. Ions with an enlarged pitch angle can be reflected by the magnetic mirror force of the magnetic structure even if they could penetrate through it without interaction with the wave. In such a case, the reflected ion population should have a parallel velocity close to the resonant velocity as determined in Equation (1) (i.e., $440-450 \mathrm{~km} \mathrm{~s}^{-1}$ ), but after reflection they will be directed antiparallel to the solar wind flow. Figure 5 shows exactly this situation. Therefore, the magnetic structure interacting with the ions trapped by the wave has a double role: first, it provides an external force that keeps the ions in the resonance condition and after the ions gained a certain amount of energy in the perpendicular direction, the magnetic force of the structure reflects those ions. This explains why these accelerated ions have a sunward direction. It should be mentioned that only those ions will be reflected by the SLAMS that have from the beginning a larger pitch angle or those that have acquired a larger pitch angle through acceleration that is enough for reflection. In this sense, the magnetic structure not only provides the resonance condition and the reflection for the accelerated (i.e., seed) ions, but also acts as a filter separating the solar wind ions with a lower pitch angle from the seed ions.

\section{CONCLUSION}

We can conclude that in our study, we provide for the first time observational evidence of an efficient acceleration mechanism in front of Earth's quasi-parallel bow shock that is capable of producing seed particles in abundance directly from solar wind particles. The GSA of ions is efficient due to the combination of two basic things: the trapping of ions by circularly polarized waves and the presence of an external force that keeps the ions in the resonant condition. Since magnetic structures and waves can be abundantly found in the foreshock region, the GSA mechanism might prove to be the primary source of seed ions for the first-order Fermi acceleration at the quasi-parallel shock. Our results do not invalidate the well known and accepted idea that scattered field aligned beam (FAB) ions might also substantially contribute to the injection process (as it has been shown by Kis et al. 2007, presenting the scattering of FAB ions). Most likely, the two mechanisms operate simultaneously, and thus they provide the first condition for an efficient firstorder Fermi acceleration of ions at the quasi-parallel bow shock. Establishing which mechanism is more important regarding the energy gain of ions involved in acceleration might be a topic of future study. However, we have to mention that Ipavich et al.
(1988) demonstrated that the percentage of alpha particles in the FAB is rather low. On the other hand, the alpha-to-proton ratio in diffuse ions is similar to the ratio that can be found in the solar wind population, as presented by Ipavich et al. (1984). Based on these previous results, this leads us to the conclusion that the scattered FAB ions are not the primary source for the injection. If this conclusion is correct, then the GSA mechanism might prove to be the most important element of the first-order Fermi acceleration of ions at the bow shock.

We thank the referee for his helpful comments and suggestions. The authors are also thankful for the support provided by the CNRS, by the Hungarian Academy of Sciences, by the French-Hungarian bilateral agreement project (TET 09-1-20100053), and by the OTKA-78674 project. We also thank Emmanuel Penou (CESR-Toulouse University/CNRS), who developed the CL software for the data display. This article is dedicated to the memory of Marksteiner Erzsebet; without her, all this would have not been possible. We express our gratitude for the high-quality data provided by the CAA (Cluster Active Archive).

\section{REFERENCES}

Axford, W. I., Leer, E., \& Skadron, G. 1978, in 10th Leningrad Seminar on Cosmophysics, Nuclear Cosmophysics, ed. G. Shaviv (Leningrad: PhysicoTechnical Institute), 125

Balogh, A., Carr, C. M., Acuña, M. H., et al. 2001, AnGeo, 19, 1207

Bell, A. R. 1978a, MNRAS, 182, 147

Bell, A. R. 1978b, MNRAS, 182, 443

Blandford, R. D., \& Ostriker, J. P. 1978, ApJL, 221, L29

Giacalone, J., Schwartz, S. J., \& Burgess, D. 1993, GeoRL, 20, 149

Gustafsson, G., André, M., Carozzi, T., et al. 2001, AnGeo, 19, 1219

Horbury, T. S., Cargill, P. J., Lucek, E. A., et al. 2001, AnGeo, 19, 1399

Ipavich, F. M., Gloeckler, G., Hamilton, D. C., Kistler, L. M., \& Gosling, J. T. 1988, GeoRL, 15, 1153

Ipavich, F. M., Gosling, J. T., \& Scholer, M. 1984, JGR, 89, 1501

Khotyaintsev, Y., Lindqvist, P.-A., Eriksson, A., \& André, M. 2010, in The Cluster Active Archive, Studying the Earth's Space Plasma Environment, ed. H. Laakso, M.G.T.T. Taylor, \& C. P. Escoubet (Berlin: Springer), 97

Kis, A., Scholer, M., Klecker, B., et al. 2007, AnGeo, 25, 785

Krymskii, G. F. 1977, SPhD, 22, 327

Kuramitsu, Y., \& Krasnoselskikh, V. 2005a, PhRvL, 94, 031102

Kuramitsu, Y., \& Krasnoselskikh, V. 2005b, JGRA, 110, 10108

Malkov, M. A., \& O'C Drury, L. 2001, RPPh, 64, 429

Peredo, M., Slavin, J. A., Mazur, E., \& Curtis, S. A. 1995, JGR, 100, 7907

Rème, H., Aoustin, C., Bosqued, J. M., et al. 2001, AnGeo, 19, 1303

Russell, C. T. 1988, AdSpR, 8, 147

Scholer, M., Fujimoto, M., \& Kucharek, H. 1992, in ESA Special Publication, Vol. 346, Study of the Solar-Terrestrial System, ed. K. Itoh, S.-I. Itoh, \& A. Fukuyama (Noordwijk, Netherlands: ESA), 59

Schwartz, S. J., \& Burgess, D. 1991, GeoRL, 18, 373 